\title{
ANTARES: COUPLING PARCS WITH CATHARE-3
}

\author{
M. Forestier ${ }^{1}$, G. Girault ${ }^{1}$, F. Jacq ${ }^{1}$ and A. Sargeni ${ }^{1 *}$ \\ ${ }^{1}$ IRSN \\ BP 17 - 92260 Fontenay-aux-Roses Cedex - France
}

Marc.forestier@irsn.fr, gregoire.girault@irsn.fr, francois.jacq@irsn.fr, antonio.sargeni@irsn.fr

\begin{abstract}
In recent years, the IRSN has launched a new project to couple the first $3 \mathrm{D}$ version of the thermal hydraulic code CATHARE-3 (system) with the 3D, neutronic nodal code PARCS (core): ANTARES (Advanced Neutronics and Thermal-hydraulic for the Analysis of the Reactor Safety). The purpose of this project is to increase the IRSN capability to couple different codes, to calculate the core power distribution in CATHARE-3 and to improve the thermal hydraulic boundaries conditions in PARCS. In this way, the IRSN diversifies its available tools to perform safety analysis with improved accuracy. The current technique usually adopted in France for the safety demonstrations is the so-called 'conservative' approach, which consists of reducing all the feedback (Doppler and moderator effects) and in modifying some physical quantities in such a way to increase a power peak in an accidental transient. For this reason, these facilities ('penalties') have been implemented in ANTARES. In this paper we will give two examples of accidental transients that can be simulated with ANTARES: a REA (Rod Ejection Accident) and an inadvertent boron dilution event.
\end{abstract}

KEYWORDS: ANTARES, REA, boron dilution, multi-physics

\section{INTRODUCTION}

In the last years CEA, EDF, Framatome and IRSN have developed the new, first 3D version of the core and system code (thermal hydraulic) CATHARE-3 [1] with the goal, at the same time, to improve the physical modeling of CATHARE-2 and the software architecture. The CATHARE-3 improvements have been essentially achieved in thermal hydraulic modeling (multi-fields, turbulence) and in the structure 3D modeling by the use of fine and not conforming, structured meshes. The internal, neutronic kinetic model, as in CATHARE-2, is remained a point kinetic model.

Since few years, the IRSN has launched a new project to couple CATHARE-3 with the 3D neutronics code PARCS [3]: ANTARES (Advanced Neutronics and Thermal hydraulic for the Analysis of the Reactor Safety). The purpose of this project is to increase the IRSN capability to couple different codes, to calculate the core power distribution in CATHARE-3 and to improve the thermal hydraulic boundary conditions in PARCS. In this way, the IRSN diversifies its available tools to perform the safety analysis that is charged to do for the French Nuclear Safety Authority (nowadays, IRSN is currently using the computational chain HEMERA.V1 [2]).

\footnotetext{
${ }^{*}$ Corresponding Author
} 
In this paper, we will give a quick description of ANTARES (section 2) and we will emphasize how CATHARE-3 and PARCS have been coupled in section 3. Sections 4 and 5 will contain two examples of accidental transients that can be simulated with ANTARES: a REA (Rod Ejection Accident) and an inadvertent boron dilution event.

\section{ANTARES}

ANTARES is developed by the IRSN in order to couple two codes: PARCS [3] (neutronics, nodal core, fed with cross section multi-parameter library by CASMO5 [4]) and CATHARE-3 [1] (core and system thermal hydraulics), with an explicit coupling. ANTARES is formed by a set of ad hoc routines that allows for the data exchange and guarantees the compatibility between the two geometries' codes. Figure 1 gives a presentation of the ANTARES structure:

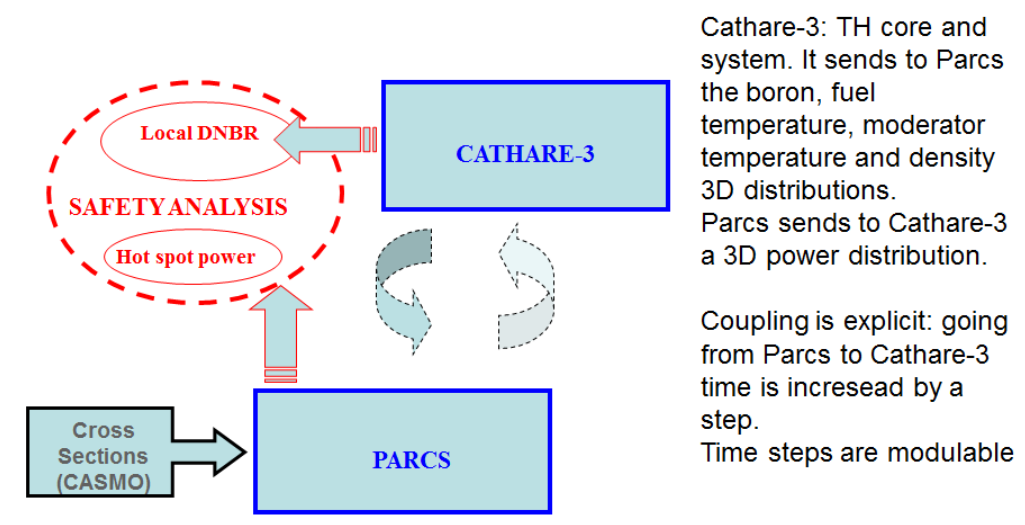

Figure 1: ANTARES structure

One channel in the thermal hydraulic geometry corresponds to one assembly.

In the following, we give an overview of the PARCS and CATHARE-3 codes which constitute the ANTARES chain.

\subsection{PARCS}

PARCS (Purdue Advanced Reactor Core Simulator, [3]) solves the 3D space-time Boltzmann equation (steady-state and time-dependent, diffusion and low-order transport), in cartesian and hexagonal geometry and in multi-group approximation. PARCS is fed in cross sections by the CASMO5 lattice code, which prepares an external cross sections library read by PARCS The cross sections model is based on multiparametric tables (the parameters are burn-up, the square root of the fuel Doppler temperature, moderator density, and temperature, boron, rod in/out). The cross sections by mesh, depending on the mesh parameter values, are computed via a linear interpolation between the library cross sections. PARCS has an important feature: it has been designed to be connected to any thermal-hydraulic system code and this has greatly facilitated the coupling with CATHARE-3.

The diffusion equation is solved using the finite differences and the nodal method (coarse meshes) with the introduction of discontinuity factors. PARCS is used in the ANTARES chain with the following options: nodal method to solve the diffusion equation; 4 nodes (meshes) by assembly and 2 energy groups. 
The IRSN is authorized to use the code PARCS in the framework of an agreement with the Nuclear Regulatory Commission (USA).

\subsection{CATHARE-3}

CATHARE-3 [1] is a system code developed by CEA, Framatome, EDF and IRSN. This code is a part of the NEPTUNE project (launched in 2001) that includes software development, research in physical modeling and numerical methods, development of advanced instrumentation techniques and new experimental programs.

In 2006 the strategy for the system scale of NEPTUNE was defined and the CATHARE-3 development was launched. The main objectives are:

- advanced physical modeling of two-phase flows, mainly by using multi-field and turbulence models,

- improved 3D modeling by the use of fine and non-conforming structured meshes,

- generalized coupling capabilities with other thermal hydraulic scales and with other disciplines (core physics, structural mechanics, ...),

- extension of the applicability to new Generation IV reactors (Sodium Cooled Fast Breeder Reactors, Gas Cooled Reactors, Supercritical Light Water Reactors),

- a true object-oriented software architecture.

At the same time CATHARE- 3 is in continuity with the CATHARE-2 code which is the current industrial version of CATHARE and internationally used for nuclear power plant safety analysis, in simulators, and coupled simulation tools.

\section{COUPLING}

The coupling has been achieved, from a theoretical point of view, defining an Application Programming Interface (API) between the generic TH code and the generic neutronic code, which makes its numerical implementation as easy as possible. The API It is based on two simple ideas: 1) each code must be split in a limited set of well-defined tasks and 2) each code must provide additional services to enable the exchange of data. Two types of services are foreseen: providing and receiving data.

Since the codes can use different geometries, the API will provide additional services to interpolate fields between geometries (a field is split - or integrated - proportionally to the fraction of the occupied mesh volumes, in such a way to conserve the original physical quantity). Tasks and services of each code will be called by an external program, the pilot, which implements the coupling scheme. The TH code is not allowed to call directly services of the neutronic code and reciprocally. This warrants rigorous programming orthogonality between the codes. All the code services consist in getting or setting pieces of information needed by the codes or the pilot itself:

- The two codes may have different meshing for the core region. Each code must be able to define its geometry. Each code will have to provide two similar services: returning first the size of its geometry and, after that, its geometry based on the previous definitions.

- Each code has to provide fields applied to its geometry. For instance, the neutronic code must return a power distribution (one value by mesh). But it must also provide the total power under the form of a scalar value.

- Each code will then have to provide four services to get (to give) and to set (to receive) scalars or fields (total power, control rods position, fuel temperature, moderator temperature and density, boron distribution). 


\section{PENALTIES}

In France, the current technique for the safety demonstration of an accidental transient is a 'conservative' approach, i.e., to facilitate the increase of the power peak by decreasing the neutronic feedback and by modifying some physical quantities. For instance, for a rod ejection, this means to increase the rod worth, to decrease the delayed-neutron fractions, the Doppler and moderator effects. All these possibilities have been implemented in ANTARES and we sketch in the following the techniques used.

\subsection{Rod Worth}

The modification of a control rod worth is obtained via a modification of the macroscopic production cross section. The basic idea is to modify the production section to have an imposed reactivity difference rod-out/rod-in without flux distortion and neglecting the feedback.

Writing the rod-out, unperturbed static equation as:

$$
(A-\lambda F) \varphi=0, \text { with the reactivity defined as: } \rho=1-\lambda
$$

If feedback are kept constant, the eigenvalue can be changed imposing:

$$
\lambda F=\lambda^{\prime} F^{\prime}, \text { where } \lambda^{\prime} \text { is the eigenvalue-goal and the new reactivity is } \rho^{\prime}=1-\lambda^{\prime}
$$

What we want to have is no modification when the rod is in (time $t_{0}$ ) and a complete modification when the rod is out (time $t_{F}$ ) and we choose to obtain it by using a time-dependent arbitrary linear function. In such a way, the new production operator becomes:

$$
F^{\prime}=\left[\left(\frac{1-\rho}{1-\rho^{\prime}}-1\right) \alpha(t)+1\right] F, \text { with } \alpha(t)=\frac{t-t_{0}}{t_{F}-t_{0}}
$$

Remark: the reactivity effects have been computed using the dynamic reactivity. In this range of reactivity variations (few hundreds of $\mathrm{pcm}$ ) the static and dynamic reactivity [6] have, practically, the same value.

\subsection{Delayed-neutrons fractions}

The modification of the delayed-neutrons fractions (the 'betas') is simply obtained by multiplying the betas' core distribution by a factor.

\subsection{Doppler and moderator effects}

A reactivity effect is defined as the difference between the reactivity of the core at a time ' $t$ ' and the steady-state reactivity, when only the value of a physical quantity is modified with respect to the steadystate values. With this definition, the Doppler/moderator effects are the reactivity effects due to the fuel temperature/moderator density. The penalty of these reactivity effects (i.e., the feedback reduction) is obtained by multiplying the fuel temperature/moderator density distributions by a coefficient in such a way to have the required reactivity effects. The technique is the following:

- Compute the reactivity effect, during the transient, at the time ' $t$ '. Subtract the penalty. The value obtained is the goal;

- Multiply the fuel temperature/moderator density by a coefficient and compute a new reactivity effect; 
- If the goal is not reached, an iterative method (as Newton method) is used to search for a new coefficient until to reach the goal.

Remark: the temperature/density distributions are not distorted, the transformation is homothetic.

\section{EXAMPLE: ROD EJECTION ACCIDENT}

The Rod Ejection Accident (REA) is a quick expulsion of a control rod out of the core (about $0.1 \mathrm{sec}$ ) that leads to a fast reactivity insertion and to a rapid increase and important deformation of the power. As a consequence of the power increase, fuel temperature increases too, and fuel rods can be damaged, resulting in a risk of radioactive material dispersion. The increase of power is limited by the neutronics feedback, Doppler and moderator effects, and completely stopped by the control rods emergency shutdown. Due to the accident dynamics, only the TH core is modeled in CATHARE-3 and not the system (we consider that inlet mass flow and moderator temperature do not change within the simulation time - one second - and pressure remains constant). In this type of accident, the time step, due to the very quick rod ejection, is set to 0.001 seconds, i.e., every 0.001 seconds a power distribution and a temperature fuel/density moderator are exchanged between PARCS and CATHARE-3.

As an example of the possibilities of ANTARES, we present here two REA transients, one Best-Estimate and another one where some penalty techniques (implemented in ANTARES) are used: the rod worth is imposed $(450 \mathrm{pcm})$; the delayed-neutron fraction is set to $420 \mathrm{pcm}$; the Doppler and moderator effects are decreased of, about, 30\% each. The modeled core is a 2750 MWth PWR (157 loaded UO2 (70\%) and MOX (30\%) fuel assemblies). The REA is initiated at 40\% Nominal Power (NP). The core irradiation corresponds to the end of a cycle.

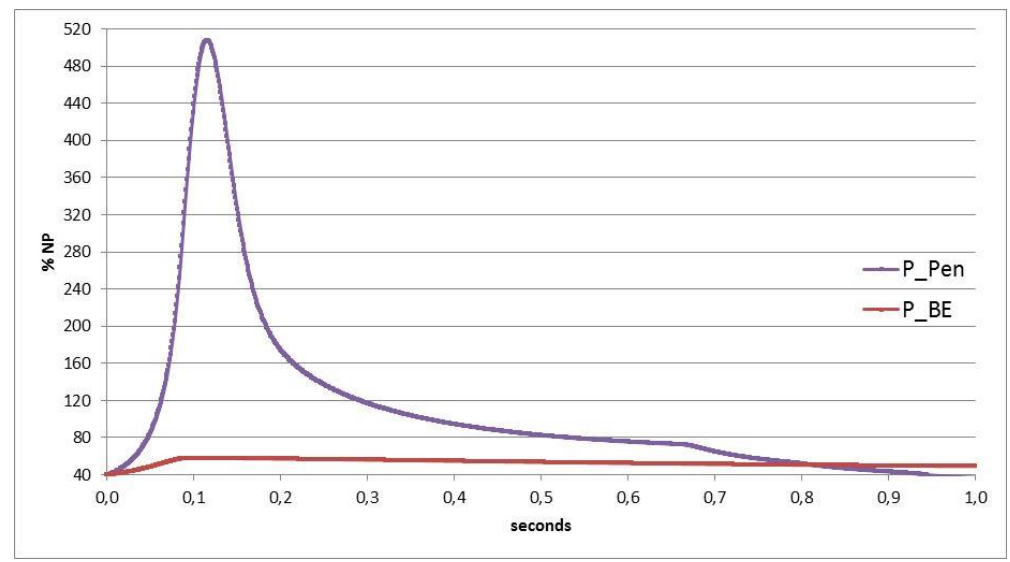

Figure 2: Power transient in case of $R E A$ with $\left(P_{-}\right.$Pen) and without penalties $\left(P_{-} B E\right)$

The penalties' effects are clearly visible. The 'penalized' power peak is a magnitude order bigger than the Best-Estimate one.

Figure 3 (zoom around the power peak) shows the contributions to the power due to the different penalties. The curve 'Antares' is with all the penalties (rod worth, beta effective -Beff-, Doppler and moderator); the curve 'A_RodWorth_Beff' is with only rod worth and beta effective (which are responsible for the more important power increase); the curve 'A_Dopp' is with rod worth, beta effective and Doppler; 'A_Moderator' is with rod worth, beta effective and moderator. The moderator penalty is not very effective for this type of transient (water warms up slowly). To decrease the Doppler effect is two times more effective to increase the power peak. 


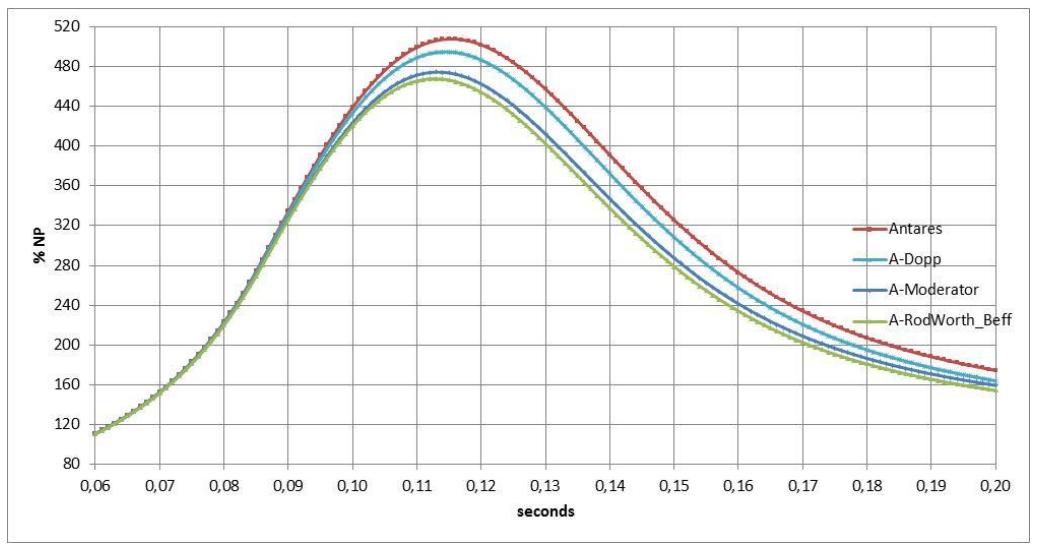

Figure 3: Rod Ejection with penalties

\section{EXAMPLE: INADVERTENT BORON DILUTION EVENT}

Boron dilution event (homogeneous or inhomogeneous) [4] can arrive by an inadvertent decrease of the boron concentration in the primary coolant. Homogeneous dilution may occur due to CVCS (Chemical Volume Control System) maloperation when the primary pumps or natural circulation are sufficient for the uniform mixing of the whole primary circuit. Inhomogeneous dilution takes place if a slug of low (or non-) borated water is formed in the primary loop. Then there is a risk of a potentially, rapid core power increase if the slug is transported without sufficient mixing to the reactor core by pumps startup or by reestablishing natural circulation. This slug could be formed by a non-borated injection of CVCS into a stagnant loop or through leakages from other circuits.

Both possibilities can be simulated in ANTARES. The technique used is to divide the computation in two steps: 1) with a CFD code the slug transport from the primary loop until the core inlet is simulated; that allows having some time-dependent boundary conditions by channel (boron, enthalpy, flow rate) and 2) ANTARES (in this case, only the core is modeled, not the whole system) runs a transient due to these time-dependent boundary conditions.

As an example (same reactor as the REA), we show a simulation of an inhomogeneous dilution computed with ANTARES. The core is simulated in an initial steady-state corresponding to an HZP (Hot Zero Power) state with pumps on, reactivity equal to $-4 \$$, all the rods in, boron concentration equal to roughly $750 \mathrm{ppm}$. A non-symmetric slug of non-borated water is injected in the core for 50 seconds; in the following 50 seconds the boron level is restored and, after, ANTARES is free to run for 75 seconds. Table 1 summarizes the transient scenario:

Table 1: Transient Scenario

\begin{tabular}{|c|c|}
\hline Time & Events \\
\hline $0-25 \mathrm{~s}$ & $\begin{array}{r}\text { Steady State Stabilization (Cathare-3 equilibrium state). Reactivity }=- \\
2000 \mathrm{pcm} \text {. Boron }=749.82 \mathrm{ppm} \text { (constant in the core) }\end{array}$ \\
\hline $25-75 \mathrm{~s}$ & $\begin{array}{r}\text { Boron linear dilution: from the nominal value }(749.82 \mathrm{ppm} \text { ) to a different } \\
\text { final value by assembly (see Figure } 2)\end{array}$ \\
\hline $75-125 \mathrm{~s}$ & Restoration of the boron nominal value \\
\hline
\end{tabular}

Figure 2 shows the core radial plane of boron values by assembly. The inhomogeneous dilution is in the North-West core quarter (numbers represent the final bore values, i.e., at $75 \mathrm{~s}$ ): 


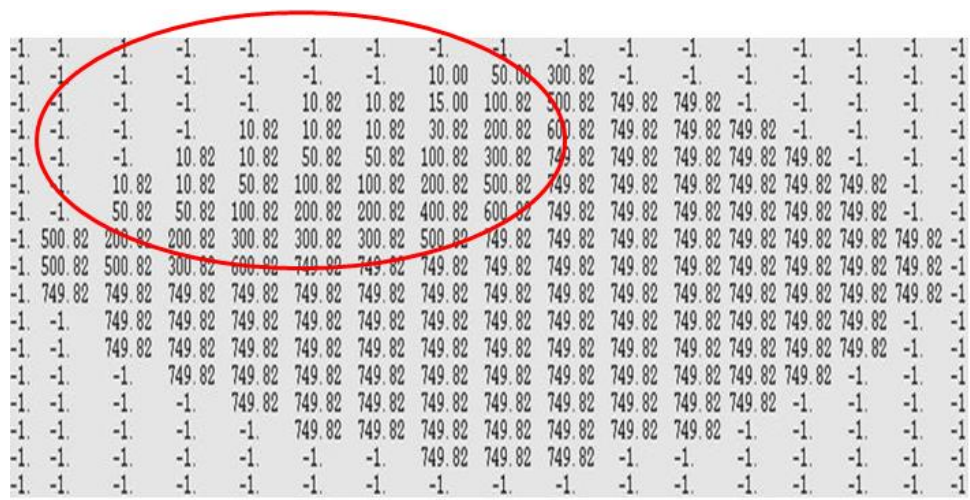

Figure 2: Inhomogeneous Dilution - Core Radial Plane

The core power is presented in Figure 3:

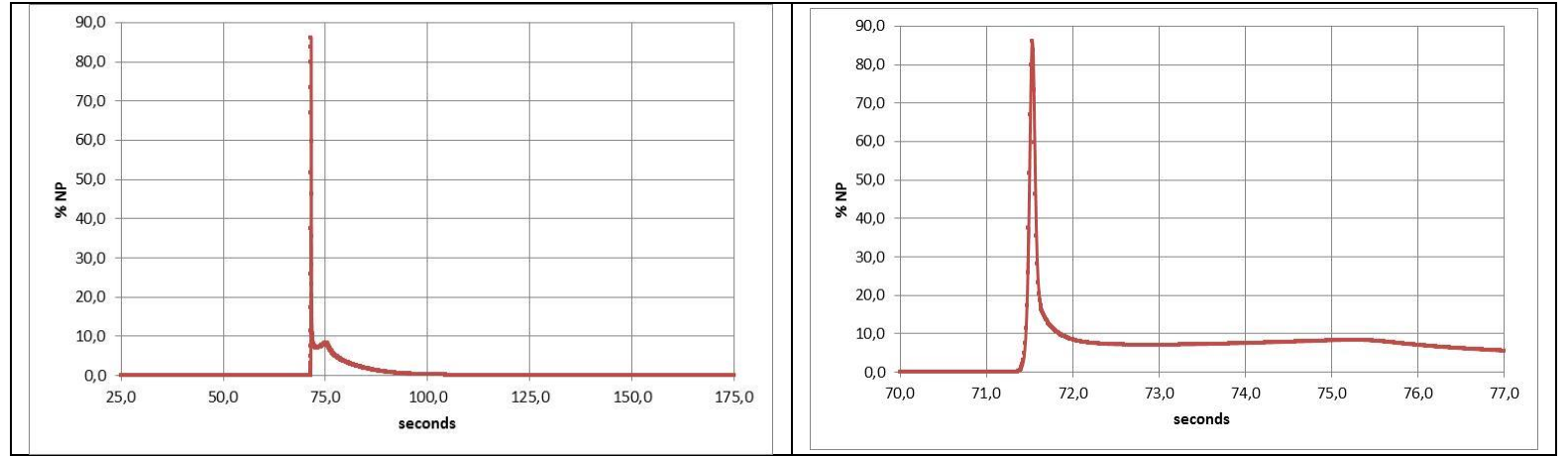

Figure 3: Inhomogeneous Dilution - Core Power (complete and zoom)

The power peak appears between 71 and 72 seconds. It decreases very quickly and it seems to have a plateau for some seconds until $75.5 \mathrm{~s}$ and, after, it decreases again due to the boron reintroduction. An interesting analysis of the physics of the transient can be done analyzing the reactivity effects. Figure 4 shows the total (blue), boron (violet), Doppler (red) and moderator (green) effects. They have been evaluated by a reactivity difference between a reactor state where only one parameter has changed compared to the steady-state.

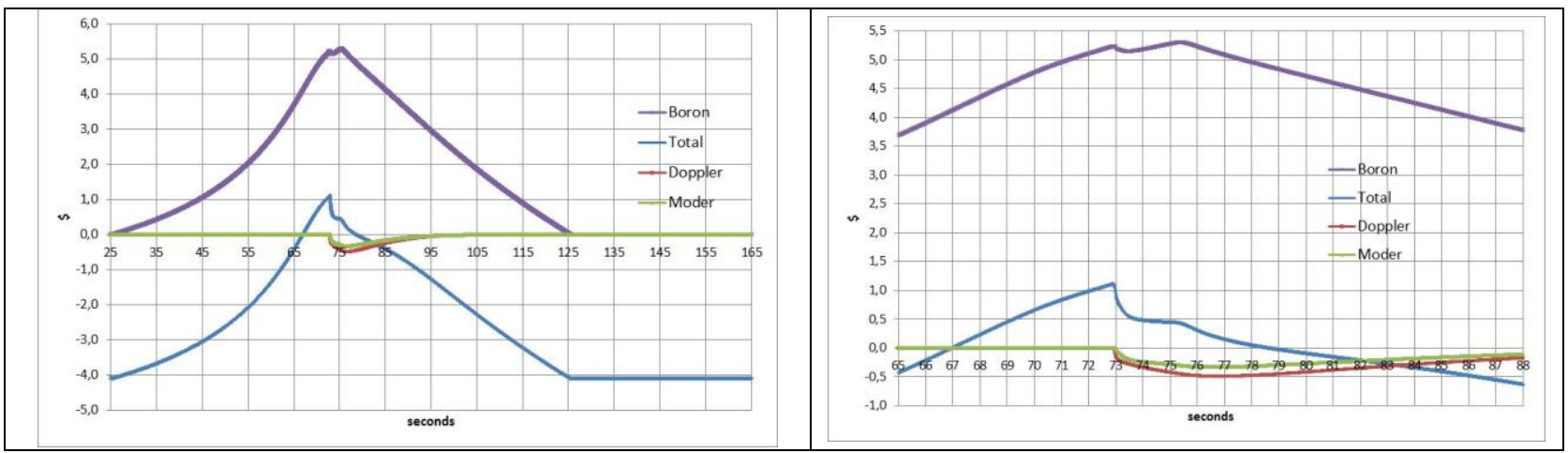

Figure 4: Inhomogeneous Dilution - Core Reactivities (complete and zoom) 
The total reactivity increases until $73 \mathrm{~s}$, when the boron reactivity decreases a bit due to the water warming (the moderator reactivity appears with a negative sign) but it starts again to increase because the boron continues to decrease. For some seconds there is compensation between the reactivity loss due to the Doppler and moderator effects and the reactivity gain due to the boron. This equilibrium allows to the power to remain nearly constant.

\section{CONCLUSIONS}

In this paper we present the development and the first results of the new computational chain ANTARES. ANTARES is the coupling (explicit) between the neutronic core code PARCS and the core and system TH code CATHARE-3. Due to the necessity to adopt a conservative approach for the safety demonstrations (following the French, current methodology), some penalties techniques have been implemented in ANTARES to maximize the power peak. Two simulations of accidental transients are shown: a rod ejection and an inadvertent boron dilution. These examples demonstrate that ANTARES can simulate these two important accidents. Work is in progress to simulate other types of accidental transients and to improve the validation of the chain using the available international numerical and experimental benchmarks

\section{NOMENCLATURE}

REA $=$ Rod Ejection Accidente; IRSN $=$ French Technical Safety Organization $;$ CEA $=$ Alternative Energies and Atomic Energy Commission; EDF $=$ Electricité de France; Framatome $=$ French Industry of Nuclear Equipments; $\mathrm{TH}=$ Thermal-Hydraulics; API = Application Programming Interface; $\mathrm{CFD}=$ Computational Fluid Dynamics

\section{ACKNOWLEDGMENTS}

The authors are indebted to Dr. A. Ward, from Michigan University, for the help all along with our work.

\section{REFERENCES}

1. P. Emonot, A. Souyri, J.L. Gandrille, F. Barré, "CATHARE-3: a new system code for thermailhydraulics in the context of the NEPTUNE project", Nuclear Engineering and Design, 241, pp. 44764481 (2011).

2. F. Dubois, B. Normand, A. Sargeni, "An example of neutronic penalizations in reactivity Transient Analysis Using 3D Coupled Chain HEMERA", Advances in Reactor Physics-Linking Research, Industry and Education (PHYSOR 2012), April 15-20, 2012, Knoxville, Tennessee, USA

3. Downar, T., Xu, Y., Seker, V., 2009. PARCS, U.S. NRC Core Neutronics Simulator, University of Michigan.

4. CASMO5: www.studsvik.com/our-solutions/products/casmo

5. IAEA, Safety Report Series No30, 'Accident Analysis for Nuclear Power Plants with Pressurized Water Reactors', 2003

6. D. Rozon, Introduction à la cinétique des réacteurs nucléaires, Ecole Polytechnique de Montréal, Canada, 1992 